\title{
Formação pedagógica na enfermagem: reflexão para a prática
}

\author{
Pedagogical education in nursing: reflection for practice
}

Formación pedagógica en enfermería: reflexión para la práctica

Rosilene da Silva ${ }^{1 *}$, Regina Racquel dos Santos Jacinto², Rogério Gomes Pereira².

\begin{abstract}
RESUMO
Objetivo: Apresentar uma reflexão para a importância da formação pedagógica para docentes de ensino superior em enfermagem. Revisão bibliográfica: A formação pedagógica de professores para o nível superior tem representado uma nova roupagem para a formação profissional, novas tecnologias têm sido utilizadas no auxílio do desenvolvimento das competências dos formadores. Estudos têm mostrado que o processo de ensino do cuidar precisa superar o modelo tradicional e tecnicista, buscar a ressignificação da prática pedagógica e romper com abordagens conservadoras, tendo em vista que cada vez mais é preciso ensinar e aprender, conceitos esses que de longe parecem fáceis, sobretudo nos tempos atuais em que os profissionais precisam ref letir sobre o seu cotidiano com maior profundidade, haja vista tantas transformações do contexto vivido, um desafio constante. Considerações finais: A literatura mostra que o profissional enfermeiro precisa ser um pesquisador da própria prática e a formação do docente enfermeiro precisa ser redirecionada na reflexão da prática cotidiana. Assim sendo considera-se a reflexão sobre a formação e expansão de cursos necessária, assim como, pertinente saber quem serão os atores que responderão pela formação e, inclusive qual a preocupação que existe para remover barreiras para a aprendizagem.
\end{abstract}

Palavras-chave: Educação em saúde, Ensino superior, Enfermagem.

\begin{abstract}
Objective: To present a reflection on the importance of pedagogical training for higher education professors in nursing. Bibliographic review: The pedagogical training of teachers for higher education has represented a new look for professional training, new technologies have been used to help develop the skills of trainers. Studies have shown that the process of teaching care needs to go beyond the traditional and technical model, seek a redefinition of pedagogical practice and break with conservative approaches, considering that it is increasingly necessary to teach and learn, concepts that seem easy from afar, especially in current times when professionals need to reflect on their daily lives in greater depth, given so many changes in the context experienced, a constant challenge. Final considerations: The literature shows that the professional nurse needs to be a researcher of their own practice and the training of the nursing teacher needs to be redirected in the reflection of daily practice. Therefore, the reflection on the training and expansion of courses is considered necessary, as well as it is pertinent to know who the actors will be who will be responsible for the training, and even what concern exists to remove barriers to learning.
\end{abstract}

Key words: Health education, Higher education, Nursing.

\section{RESUMEN}

Objetivo: Presentar una reflexión sobre la importancia de la formación pedagógica para profesores de educación superior en enfermería. Revisión bibliográfica: La formación pedagógica de los profesores de

1 Universidade Brasil, São Paulo - SP. *E-mail: rosilenesilva2005@yahoo.com.br

2 Universidade Federal do Maranhão, São Luís - MA.

SUBMETIDO EM: 10/2021

ACEITO EM: 10/2021

PUBLICADO EM: 11/2021

REAEnf | Vol. 15 | DOI: https://doi.org/10.25248/REAEnf.e9080.2021 
educación superior ha representado una nueva mirada para la formación profesional, se han utilizado nuevas tecnologías para ayudar a desarrollar las habilidades de los formadores. Los estudios han demostrado que el proceso de enseñanza del cuidado necesita ir más allá del modelo tradicional y técnico, buscar una redefinición de la práctica pedagógica y romper con enfoques conservadores, considerando que cada vez es más necesario enseñar y aprender, conceptos que parecen fáciles desde lejos. especialmente en los tiempos actuales en los que los profesionales necesitan reflexionar con mayor profundidad sobre su día a día, ante tantos cambios en el contexto vivido, un desafío constante. Consideraciones finales: La literatura muestra que el profesional de enfermería debe ser investigador de su propia práctica y la formación del docente de enfermería debe reorientarse en la reflexión de la práctica diaria. Por ello, se considera necesaria la reflexión sobre la formación y ampliación de cursos, así como, es pertinente conocer quiénes serán los actores que se encargarán de la formación, e incluso qué preocupación existe por remover barreras al aprendizaje.

Palabras clave: Educación para la salud, Educación superior, Enfermería.

\section{INTRODUÇÃO}

Em tempos atuais ressalta-se uma fértil discussão sobre educação e formação, ratificada pelas rápidas transformações das sociedades vistas na era em que vivemos, a competitividade e a luta pelo domínio do saber, têm feito suscitar muitas questões, sobretudo a respeito da força de trabalho de enfermagem e sua importância no contexto em que se tornou o ator principal, protagonista do cuidado ao paciente, de forma corajosa e ef etiva e ininterrupta na pandemia de Covid-19 (OLIVEIRA APC, et al., 2020).

A formação pedagógica para docentes na área da saúde, tem se tornado cada vez mais necessária à prática de um cuidado seguro, e define as condições para o exercício da atividade no âmbito da formação inserida no mercado de trabalho, na atual conjuntura, de grande importância na superação de problemas crônicos e emergentes na formação (WORLD HEALTH ORGANIZATION (WHO), 2020).

As exigências do mercado de emprego atual, têm feito com que os profissionais se dediquem mais, no sentido de criarem condições de ensino-aprendizagem, agindo de forma a buscar a qualidade do aprendizado, soluções formativas de qualidade, flexíveis e personalizadas; referenciais pedagógicos são necessários na formação em enfermagem (LIMA MM, et al., 2017). A grande maioria dos professores de hoje, na nossa visão, já entendeu que aquela figura prepotente, que acha que domina o conhecimento, traduz uma atitude arcaica e surrealista e que não mais se ajustam às necessidades atuais (KLOH D, et al., 2014).

No ensino superior em saúde, a preocupação dos profissionais com a prática assistencial aumentou muito nos últimos 50 anos, e essa evolução tem sido facilitada pelo crescimento da produção teórica e de pesquisas; pelas mudanças no paradigma de saúde, cada vez mais é preciso ensinar e aprender, conceitos esses que de longe parecem fáceis, é preciso entender que o ensino envolve teoria e prática e, obviamente pessoas dotadas de conhecimentos prévios, acumulados ao longo de sua profissão, preocupação está voltada pela implementação de políticas públicas no enfrentamento dos desafios existentes (WHO, 2020).

As raízes históricas da enfermagem mostram um distanciamento do cuidado na enfatização dos aspectos biológicos e terapêuticos, por pressão dos interesses capitalista, a busca por reconhecimento seguiu norteando as ações de direcionamento do cuidado, ensinar e cuidar tornou-se um desafio necessário ao longo do tempo (HERTEL VL, 2020).

No que tange as atividades inerentes à profissão, no cotidiano de trabalho de enfermagem, tem sido permeadas entre a assistência, gerência, educação e pesquisa, e por isso tem sido considerada como a espinha dorsal do sistema de saúde em todo o mundo; premissas essas que essenciais à prática segura do cuidado de enfermagem, contudo, é notório com o passar do tempo, que apesar das dificuldades apresentadas no processo de ensino e/ou educação em saúde ser mais evidentes na atuação enquanto professor, estas surgem também, na sua prática assistencial, partindo-se do princípio de que cuidar e educar são indissociáveis (RIBEIRO VMB, 2011). 
Reconhecendo a importância desta articulação, pretendeu-se destacar a função do ensino pedagógico e sua importância no ensino superior em enfermagem, propondo uma reflexão acerca do exercício na prática profissional, buscando embasamento para o processo ensino-aprendizagem que promova a autonomia e a contextualização dos diversos saberes disciplinares, integrando os conhecimentos pedagógicos na prática docente de enfermagem.

\section{REVISÃO BIBLIOGRÁFICA}

Notoriamente é sabido que o desenvolvimento de competências para uso de evidências na prática envolve mudanças de valores e de prioridades, de hábitos e de rotinas, criação de bases para ir além de nossas limitações, tanto no indivíduo como na instituição, nos dias de hoje, de fato um desafio para educadores e para gestores no desenvolvimento de novos processos e prioridades, sobretudo, nesse enquadre de crise mundial (WHO, 2020).

A Organização das Nações Unidas para a Educação, a Ciência e a Cultura (UNESCO) estabelecem que quatro pilares devem sustentar a Educação do século XXI: aprender a conhecer; aprender a fazer; aprender a viver juntos; aprender a ser; vias estas que não podem ser dissociadas (UNESCO, 2012; DELORS J, 2012). Entende-se, naturalmente, que o processo de aprendizagem faz parte das condições e necessidades dos seres vivos; crescemos, evoluímos e o aprendizado nos acompanha; aprendemos em cada gesto, em cada vivência, em cada situação; e diante dos desafios do século XXI, de transição demográfica acelerada, a aprendizagem ao longo da vida é uma consequência necessária e de grande importância para a formação holística dos seres humanos (LOPES-JÚNIOR LC, 2021).

A aprendizagem faz parte das nossas vidas, fundamental para nossa evolução; começamos a aprender em casa com nossa família, informalmente: a comunicar, a andar, a falar, a saltar, a andar de bicicleta, a amar, a respeitar, a valorizar a família e seus princípios. Crescemos e a escola nos traz o conhecimento formal, um mundo de conhecimentos que nos situam no mundo, conciliados ao que aprendemos em casa, adquirimos comportamentos no meio ambiente e na sociedade, descobrimos que a curiosidade, os questionamentos, a motivação, as aptidões, as ações e reflexões são contextos estimulantes que nos garantem sucesso pessoal e social, aprendizagem é sempre uma construção pessoal, um processo complexo que não se faz sozinho, precisamos do outro, e assim também aprendemos enquanto ensinamos. O feedback entre aluno e professor é essencial, a troca de experiências e conhecimentos norteiam a formação do aluno, além disso a formação do professor (CARDOSO ACS, 2018).

O ensino pode ocorrer através de uma atividade comunicativa, ou seja, ensinamos quando partilhamos, orientamos ou informamos, é preciso que haja uma prática de preparação para a socialização no exercício de ensinar, é preciso compreender que esse é um processo complexo que envolve relação, motivação dos intervenientes, conteúdos propostos, códigos utilizados e concepções que os agentes têm dessa interação (KLOH D, et al., 2014). Quando pensamos na enfermagem vimos que esse processo ainda tem muito o que ser revisto, ainda observamos muitas dificuldades na efetividade de projetos pedagógicos, notamos dificuldades na comunicação específica entre equipes, e compreendemos que é preciso buscar o distanciamento da visão tecnicista (LIMA MM, et al., 2017).

Quanto mais vivemos, maior quantidade de experiência acumulamos ao longo da vida, e, este enfoque, deve ser muito bem analisado, principalmente na área da Saúde, visto que é notório a pluralidade de conhecimentos diversos e muitas vezes se torna mais complexo o distanciamento, por se tratar de profissionais com experiências acumuladas, são seres pensantes, o enfoque na integralidade do cuidado, princípio do Sistema Único de Saúde (SUS), onde se busca entender a pessoa como um todo indivisível e inserido num contexto social, familiar e cultural, deveria ser evidenciado na relação pedagógica de forma enfática, a visão holística deve permear o cuidado do enfermeiro (KLOH D, et al., 2015).

A literatura nos mostra que o professor deve ser o mediador da aprendizagem, elaborando uma comunicação horizontal, buscando o desenvolvimento pessoal, construindo espaços para o envolvimento de todos e, assim tornar a aprendizagem uma troca de conhecimentos, compartilha de experiências vivenciadas. 
Este conceito deveria ser imprescindível, certamente crucial no processo de aprendizagem. Da mesma forma o aluno, por sua vez, tem um papel importante nesse processo, o de buscar o conhecimento de forma proativa, orientar a sua vontade de aprender, tendo em conta as suas potencialidades e aptidões, construir a sua estrutura do saber e do saber fazer. O processo de educação é marcado pela inter-relação professor-aluno e aluno-professor, prática do feedback na busca de uma motivação para a aprendizagem a longo prazo (FARIAS CML, 2015; CARDOSO ACS, 2018).

A formação pedagógica de professores para o nível superior visa buscar uma nova roupagem para a formação profissional, novas tecnologias no auxílio do desenvolvimento das competências dos formadores no intuito de melhorar a qualidade da formação profissional, dessa forma adotando novos recursos de aprendizagem com os avanços dos computadores e das comunicações via satélite e é relevante que essa formação seja inerente à prática cotidiana, não apenas na formação inicial, mas também de forma continuada (KLOH D, et al., 2015).

Neste contexto estudos mostram que a adoção do modelo de andragogia, é importante e mais apropriado para o ensino de adultos, e vale lembrar ao exemplificarmos a saúde, a experiência adquirida constitui um recurso importantíssimo e, precisa ser valorizado e potencializado. Contrariamente, no modelo pedagógico, essa experiência é considerada de pouca utilidade, para suas próprias aprendizagens, ou seja, o método pedagógico está interessado em simplesmente passar o conteúdo a diante e, o andragógico preocupa-se em facilitar a percepção do conteúdo aos alunos, a aprendizagem está centrada no aluno que poderá fazer a aplicação desse conteúdo na prática na vida diária (BARROS R, 2018).

No ensino do cuidar, algumas práticas pedagógicas precisam ser compreendidas, a tendência tradicional, em que prevalece a fala do professor, ainda presente nas escolas de enfermagem, a tendência tecnicista, em que se atua de forma a comprometer a visão do todo, age-se de forma mecânica, automática, repetitiva e programada, e impedindo assim as ações reflexivas e críticas; e a tendência libertária em que se pontua a educação reflexiva e libertadora, o aluno passa a ser valorizado, compreendido e passa a fazer parte do ensino-aprendizagem numa relação horizontal entre educando e educador (SIMÃO A, 2013). O processo de ensino do cuidar precisa superar o modelo tradicional e tecnicista, é preciso buscar a ressignificação da prática pedagógica, rompercom abordagens conservadoras, estar aberto a mudanças, à reflexão-ação, evitando o distanciamento entre teoria e prática (HERTEL VL, 2020).

De acordo com a Resolução CNE/CES nํ. 3, 2001 do Ministério da Educação, as Diretrizes Curriculares Nacionais do Curso de Enfermagem delineiam a formação do enfermeiro, direcionando a atuação do enfermeiro para as competências e habilidades na atenção à saúde. Apesar dos profissionais já possuírem preparo nas suas áreas de especialidade, é nítido o despreparo como docente, e na era globalizada em que estamos inseridos, padrões de excelência estão cada dia mais em destaque na busca de uma identidade profissional para a docência de ensino superior (MENDES IAC, et al., 2020).

Nesse sentido verificamos que continuamos enfrentando um enorme desafio: o de formar profissionais de saúde, diante das grandes mudanças que têm nos acompanhado, dias desafiadores, além da era tecnológica do século XXI, que avança a passos gigantes e num ritmo acelerado; formarprof issionais voltados para a integralidade do cuidado é cada vez mais um grande desafio, o que nos traz também, consequentemente oportunidade de crescimento e desenvolvimento (TARDIF M, 2012).

Diante do cenário da enfermagem no Brasil, inúmeros são os fatores políticos, sociais e econômicos que conduziram a trajetória da enfermagem no Brasil e isso tem se refletido diretamente na sua prática e na busca por uma formação permanente e de qualidade. A busca constante pelo conhecimento de didática vem aumentando gradativamente, e assim conduzir a indagações, cada vez mais necessárias, que tencionam a implementação de uma prática docente capaz de unir teoria à prática, com técnicas educacionais atuais e envolventes, formando novos enfermeiros com pensamento crítico reflexivo sobre seu exercício profissional e na edificação de uma sociedade responsável (LIMA MM, 2017).

Diante da complexidade da prática assistencial em que as especificidades do processo ensinoaprendizado necessitam ser discutidas e contempladas em todas as suas dimensões, ensino, aprendizagem 
e assistência, estudos pontuam que ainda para muitos professores a docência em saúde é considerada secundária. Compreender e avaliar cada cenário num esforço coletivo é um contributo grandioso, e numa outra dimensão, é necessário contributo à educação desses profissionais, é necessário oportunidade de discussão dos entraves no ensino; é necessário não se limitar à visão tecnicista; é necessário redefinição dos processos de formação (FONTES FLL, et al., 2019; LIMA MM, et al., 2017).

O crescimento global das instituições fez com que ampliasse os debates sobre os impactos nas formações de ensino superior, sobretudo na saúde, crescimento este exponencial na área da enfermagem, o que traz alertas para as devidas qualificações, o CENSO da educação superior mostra um aumento do número de professores do ensino superior, incluindo a enfermagem, que adicionalmente passou a incluir também o ensino à distância, notoriamente, é sabido que o número aumentado de professores, ou de cursos, não garante a formação de docentes aptos à prática pedagógica, portanto, é importante que não se negligencie as práticas didáticas-pedagógicas (INSTITUTO NACIONAL DE ESTUDOS E PESQUISAS EDUCACIONAIS ANÍSIO TEIXEIRA (INEP), 2019; MANTOVANI ICA e CANAN SR, 2016; QUINTANILHA LF, et al., 2020).

Nesse contexto, diante dessas evidências, e considerando que a enfermagem é a maior categoria no setor saúde, e portanto possui uma força de trabalho intrinsicamente vinculada à consequências econômicas, sociais, políticas, é preciso repensar a proliferação de novos cursos de graduação, tendo em vista que nos últimos anos o ensino superior em saúde tem sido expandido de forma célere, é necessário avaliar o grau de envolvimento dos professores na busca de formar profissionais qualificados, críticos e reflexivos; isso pode exigir reformulação das práticas pedagógicas pautada no conhecimento científico e voltados para a prática cotidiana (PÜSCHEL VAA, et al., 2017; COELHO MP, 2020).

Cabe-nos a despeito, interrogar que profissionais queremos formar? Estudos nos mostram que a Ciência do século XXI direciona nossas atitudes, nossos pensamentos no sentido de adequação às mudanças advindas da atualidade. Questionamentos diários deveriam estar presentes, uma vez que sem dúvidas queremos formar profissionais com senso crítico que adote novas formas de pensamento, e cuja competência técnica seja norteado pela humildade e chance de voltar ou rever procedimentos, erros; capazes de questionamentos diários, busca constante pela recriação da prática cotidiana, voltada para a realidade e assim contribuindo para a sua transformação; profissionais sobretudo comprometidos e engajados com a promoção em saúde e que entenda o ser humano com um ser holístico (SILVA RRD, et al., 2021).

Por fim, ratifica-se que a situação da enfermagem no mundo está sob discussões, diante do cenário de saúde em que vivemos, o cuidado tem permeado discussões e a busca pela valorização da profissão nunca se fez tão presente, nota-se o fornecimento de bases para conversas no âmbito global, que tem suscitado valorização da profissão; o entendimento é amplo de que este momento é crucial para a enfermagem em todo o mundo, é imperativo que a voz da enfermagem em todo o mundo, reverbere e traga impactos que colabore com a transformação do presente, que vise, além de tudo, ao dinamismo do processo ensino-aprendizagem; práticas pedagógicas voltadas para o processo de aprendizagem do profissional enf ermeiro, assim como na busca pelo crescimento do corpo docente, atrelados a busca constante de formação continuada, necessárias para atualização constante do seu saber, do seu ensinar, do seu conhecimento (WHO, 2020; LOPES-JÚNIOR LC, 2021; SILVA RRD, et al., 2021).

\section{CONSIDERAÇÕES FINAIS}

Diante do exposto consideramos que é imprescindível saber quem serão os autores da aprendizagem, os alunos e os professores os quais responderão por essa formação e, sobretudo, em que condições farão isso, inclusive qual a preocupação que existe para remover barreiras para a aprendizagem, o conhecimento do aluno no final do curso e, não simplesmente a emissão de um diploma. Esta reflexão pode ser de grande relevância, contribuindo para a compreensão do cenário e da perspectiva profissional dos enfermeiros, que têm oportunidade de desenvolver seus próprios destinos, buscando um futuro em que o crescimento da produção teórica e das pesquisas em saúde tem possibilitado, visto que o cuidado prestado ao paciente está sob sua responsabilidade.

REAEnf | Vol. 15 | DOI: https://doi.org/10.25248/REAEnf.e9080.2021 


\section{REFERÊNCIAS}

1. BARROS R. Revisitando Knowles e Freire: Andragogia versus pedagogia, ou O dialógico como essência da mediação sociopedagógica. Educação e Pesquisa [en linea], 2018, 44:1-19.

2. CARDOSO ACS. O feedback aluno-aluno em um ambiente virtual de aprendizagem. Trabalhos em Linguística Aplicada, 2018;57(1): 383-409.

3. COELHO MP. Desafios na formação de enfermeiros na perspectiva dos egressos/Challenges in training nurses from the perspective of graduates. Braz. J. Hea. Rev., 2020; 3(5):13274-13291.

4. DELORS J. Educação um tesouro a descobrir - Relatório para a Unesco da Comissão Internacional sobre Educação para o Século XXI. 7ª ed. São Paulo:Editora Cortez, 2012; 281p.

5. FARIAS CML. Feedback no processo de aprendizagem: percepção dos estudantes de Odontologia em uma universidade brasileira. Revista da ABENO, 2015;15(3): 35-42.

6. FONTES FLL, et al. Desafios e dificuldades enfrentadas pelo enfermeiro docente para o exercício da docência no ensino superior. Revista Eletrônica Acervo Saúde / Electronic Journal Collection Health, 2019; Sup. 24 : e300.

7. HERTEL VL. Práticas pedagógicas na formação profissional do enfermeiro: o processo de ensino do cuidar em enfermagem. Saúde e Biociência, 2020;1(2).

8. INSTITUTO NACIONAL DE ESTUDOS E PESQUISAS EDUCACIONAIS ANÍSIO TEIXEIRA (INEP). Censo da Educação Superior 2018: notas estatísticas. 2019. Disponível em: https://download.inep.gov.br/educacao_superior/censo_superior/documentos/2019/censo_da_educacao_superior_2 018-notas_estatisticas.pdf. Acessado em 1 de outubro de 2021.

9. $\mathrm{KLOH} \mathrm{D,} \mathrm{et} \mathrm{al.} \mathrm{The} \mathrm{principle} \mathrm{of} \mathrm{integrality} \mathrm{of} \mathrm{care} \mathrm{in} \mathrm{the} \mathrm{political-pedagogical} \mathrm{projects} \mathrm{of} \mathrm{nursing} \mathrm{programs.} \mathrm{Rev} \mathrm{Latino}$ Am Enfermagem, 2014;22(4):693-700.

10. $\mathrm{KLOH} \mathrm{D,} \mathrm{et} \mathrm{al.} \mathrm{Changes} \mathrm{in} \mathrm{nurses} \mathrm{training} \mathrm{under} \mathrm{the} \mathrm{axis} \mathrm{of} \mathrm{comprehensive} \mathrm{care:} \mathrm{integrative} \mathrm{review.} \mathrm{J} \mathrm{Nurs} \mathrm{UFPE}$ Online, 2015; 9(Suppl 1): 475-83.

11. LIMA MM, et al. Indications of comprehensiveness in the pedagogical relationship: a design to be constructed in nursing education. Rev Esc Enferm USP, 2017;27(51): e03277.

12. LOPES-JÚNIOR LC. Advanced Practice Nursing and the Expansion of the Role of Nurses in Primary Health Care in the Americas. SAGE Open Nurs., 2021; 7: 23779608211019491.

13. MANTOVANI ICA, CANAN SR. Política de formação para professores do ensino superior e qualidade de ensino: um estudo sobre o programa pedagogia universitária como possibilidade de qualificação docente na perspectiva pedagógico-didática. Revista Internacional de Educação Superior, 2016;1(2): 136-148.

14. MENDES IAC, et al. Enfermagem agora e sempre: evidências para a implementação da Campanha Enfermagem Agora. Revista Latino-Americana de Enfermagem, 2020;28: e3388.

15. MINISTÉRIO DA EDUCAÇÃO. Resolução CNE/CES no․ 3, de 7/11/2001. Institui Diretrizes Curriculares Nacionais do Curso de Graduação em Enfermagem. Brasil, 2001. Disponível em: http://portal.mec.gov.br/cne/arquivos/pdf/CES03.pdf. Acessado em 1 de outubro de 2021.

16. OLIVEIRA APC, et al. O Estado da Enfermagem no Brasil. Revista Latino-Americana De Enfermagem, 2020; 28 : e3404.

17. ORGANIZAÇÃO DAS NAÇÕES UNIDAS PARA A EDUCAÇÃO, A CIÊNCIA E A CULTURA (UNESCO). Programa Mundial para a Educação em Direitos Humanos: Plano de Ação. 2012. Disponível em: https://pt.unesco.org/fieldoffice/brasilia. Acessado em 1 de outubro de 2021.

18. PÜSCHEL VAA, et al. Nurses in the labor market:professional insertion, competencies and skills. Rev Bras Enferm. 2017;70(6): 1220-6.

19. QUINTANILHA LF, et al. Formação e envolvimento pedagógico entre docentes do ensino superior em saúde: uma análise dos cursos médicos. Revista Internacional de Educação Superior, 2020; 7: e021026.

20. RIBEIRO VMB. Formação pedagógica de preceptores do ensino em saúde. Juiz de Fora/ MG: UFJF, $2011 ; 126 p$.

21. SIMÃO A. O tecnicismo pedagógico e o professor reflexivo: convergências e divergências da prática pedagógica docente. In: Congresso Nacional de Educação, XI., 2013.

22. SILVA RRD et al. Desafios da educação permanente na atenção primária í saúde: uma revisão integrativa. Saúde Coletiva (Barueri). 2021, 11(65): 6324-6333.

23. TARDIF M. Saberes docentes e formação profissional. 13a ed. Petrópolis, RJ: Vozes, 2012.325p.

24. WORLD HEALTH ORGANIZATION (WHO). State of the world's nursing 2020: investing in education, jobs and leadership. 2019. Disponível em: https://apps.who.int/iris/handle/10665/331677. Acessado em 1 de outubro de 2021. 